\title{
CHERN-SIMONS THEORY ENCODED ON A SPIN CHAIN
}

\author{
DAVID PÉREZ-GARCÍA AND MIGUEL TIERZ
}

\begin{abstract}
We construct a 1d spin chain Hamiltonian with generic interactions and prove that the thermal correlation functions of the model admit an explicit random matrix representation. As an application of the result, we show how the observables of $U(N)$ Chern-Simons theory on $S^{3}$ can be reproduced with the thermal correlation functions of the $1 \mathrm{~d}$ spin chain, which is of the XX type, with a suitable choice of exponentially decaying interactions between infinitely many neighbours. We show that for this model, the correlation functions of the spin chain at a finite temperature $\beta=1$ give the Chern-Simons partition function, quantum dimensions and the full topological $S$-matrix.
\end{abstract}

\section{INTRODUCTION}

The study of topological field theories has been a subject of continued interest in the last decades (see [1] for an early review). Chief among topological field theories is Chern-Simons theory, due to its relevance in the study of three dimensional topology and in knot theory [2], its role as the effective field theory of the fractional quantum Hall effect [3] and, more recently, its appearance also in the study of topological quantum computation [4, 5, 6] and in the description of topological strings [7].

In recent years, motivated by the realm of quantum computation, a number of proposals have been devised to simulate different quantum field theories (QFT), including non Abelian gauge theories [8]-[15]. In a somehow related but different direction, in [16], we introduced a connection, illustrated for the case of low-energy QCD, between some QFT and 1D spin systems, based on the existence of a random matrix description. This allowed us to relate crucial properties of the QFT with physically meaningful properties of the 1D system.

In this paper, following that path, we introduce a new spin chain Hamiltonian, expected to be in the same universality class as the XX-Hamiltonian, whose thermal correlation functions correspond exactly with the main observables of Chern-Simons theory, including knot polynomials. We will also discuss how a finite chain approximates these observables with an exponentially small error in the size of the chain and how finite chains models may describe Chern-Simons theory coupled with matter in the fundamental representation.

Let us first remind the basics of Chern-Simons theory, which is a three dimensional gauge theory with a simply connected and compact non-Abelian Lie group $G$ and the Chern-Simons action, given by [2]

$$
S_{\mathrm{CS}}(A)=\frac{k}{4 \pi} \int_{M} \operatorname{Tr}\left(A \wedge d A+\frac{2}{3} A \wedge A \wedge A\right),
$$

where $\operatorname{Tr}$ is the trace in the fundamental representation, $A$ is the connection, a 1-form valued on the corresponding Lie algebra, and $k \in \mathbb{Z}$ is the level. The manifold $M$ is a three dimensional compact manifold which, in this work, will be chosen to be $S^{3}$. The $q$-parameter of Chern-Simons theory is defined in terms of the level $k$ by $q=\exp (2 \pi i /(k+h))$ where $h$ is the dual Coxeter number of the Lie algebra, which is just $h=N$ in this paper since we choose $G=U(N)$. In the random matrix theory description of Chern-Simons theory that we will employ, $q$ is treated as a real number $q=\exp (-\gamma)$ (with $\gamma>0$ ) and the Chern-Simons results are recovered at the end 
by making the identification $\gamma=2 \pi i /(k+h)$. The original study of knot polynomial invariants by Jones also considers $q$ real [17] although it is possible to extend it to the case of roots of unity, in which case the necessary representation theory of the Hecke algebra is more delicate to deal with.

Let us introduce now a succinct reminder of the solution of Chern-Simons theory. In [2], a concrete description of the (finite-dimensional) Hilbert space of the theory was given: it is the space of conformal blocks of a Wess-Zumino-Witten (WZW) model [18] on $\Sigma$ with gauge group $G$ and level $k$. Here $\Sigma$ denotes the Riemann surface which is the common boundary in the Heegard splitting of the manifold $M\left[19\right.$. In the case studied here, $M=S^{3}$, the Riemann surface is a torus and for $\Sigma=T^{2}$, the space of conformal blocks is in one to one correspondence with the integrable representations of the affine Lie algebra associated to $G$ at level $k$ [2, 7]. We recall that a representation given by a highest weight $\Lambda$ is integrable if $\rho+\Lambda$, where $\rho$ denotes the Weyl vector, is in the fundamental chamber $\mathcal{F}_{l}$, with $l=k+N$ in our case. Definitions of the Weyl vector, fundamental chamber and all the other necessary concepts in representation theory are given in the Appendix, see [18] for a textbook treatment.

The states in the Hilbert state of the torus $\mathcal{H}\left(T^{2}\right)$ are denoted by $|\rho+\Lambda\rangle$ and, as we have just explained, $\rho+\Lambda \in \mathcal{F}_{l}$, since it is an integrable representation of the WZW model at level $k$. We denote these states by $|\lambda\rangle$ where $\lambda$ is the representation associated to $\Lambda$. This association is also made explicit in the Appendix. The state $|\rho\rangle$ is denoted by $|0\rangle$ and the states can be chosen to be orthonormal [2, 7] and therefore $\left\langle\lambda^{\prime} \mid \lambda\right\rangle=\delta_{\lambda, \lambda^{\prime}}$.

In addition, there is a special class of homeomorphisms of $T^{2}$ that have a simple expression as operators in $\mathcal{H}\left(T^{2}\right)$ : the $S$ and $T$ transformations, which are the generators of the $S L(2, \mathbb{Z})$ group. The matrix elements of these transformations, $T_{p p^{\prime}}$ and $S_{p p^{\prime}}$, have a very explicit expression in the basis of the integrable representations, but these will not be needed here. What is relevant here is that this non-perturbative exact solution of Chern-Simons theory computes the observables of the theory, giving [2, 7]

$$
Z\left(S^{3}\right)=\langle 0|S| 0\rangle=S_{00}
$$

This is generalized to obtain the result of the path integral in $S^{3}$ with the insertion of knots and links. Considering a solid torus where a Wilson line in representation $\lambda$ has been inserted (by inserting a Wilson loop $\mathcal{O}_{\lambda}=\operatorname{Tr}_{\lambda} U$ in the representation $\lambda$ in the path integral), then the corresponding state is $|\lambda\rangle$. Gluing this to an empty solid torus after an $\mathrm{S}$ transformation, one obtains the unknot on $S^{3}$

$$
Z\left(S^{3}, \mathcal{O}_{\lambda}\right)=\langle 0|S| \lambda\rangle=S_{0 \lambda} .
$$

The normalization of (1.2) is the quantum dimension of the representation

$$
\operatorname{dim}_{q} \lambda=\frac{\langle 0|S| \lambda\rangle}{\langle 0|S| 0\rangle}=\frac{S_{0 \lambda}}{S_{00}} .
$$

The link formed by two unknots in representations $\lambda$ and $\mu$ is the Hopf link, given by

$$
W_{\mu \lambda}=\frac{\langle\mu|S| \lambda\rangle}{\langle 0|S| 0\rangle}=\frac{S_{\mu \lambda}}{S_{00}} .
$$

These observables are (quantum) topological invariants of the manifold [20] and have an important interpretation in terms of the properties (fusion and braiding) of the quasiparticles of a topological quantum field theory. In particular, the elements of the S-matrix are closely related to quasiparticle braiding since $S_{a b}$ is equal to the amplitude for creating $a \bar{a}$ and $b \bar{b}$ pairs, braiding $a$ and $b$, and annihilating again in pair. In addition, as shown by Verlinde's formula, the $S$-matrix not only contains information about braiding, but also about fusion [4]. The quantum 
dimension of an anyonic species is a measure for the effective number of degrees of freedom of the internal Hilbert space of the corresponding particle type [21].

1.1. The two main results. The first main result of this work is an integral representation of the random matrix type, for the thermal correlation function, defined as:

$$
F_{j_{1}, \ldots, j_{K} ; l_{1}, \ldots, l_{K}}(\beta)=\left\langle\Uparrow\left|\sigma_{j_{1}}^{+} \cdots \sigma_{j_{K}}^{+} \mathrm{e}^{-\beta \hat{H}} \sigma_{l_{1}}^{-} \cdots \sigma_{l_{K}}^{-}\right| \Uparrow\right\rangle .
$$

where $|\Uparrow\rangle$ denotes a ferromagnetic state, characterized by having all of its spins up $|\Uparrow\rangle=\otimes_{i}|\uparrow\rangle$ which satisfies $\sigma_{k}^{+}|\Uparrow\rangle=0$ for all $k$, and the state is also normalized $\langle\Uparrow \mid \Uparrow\rangle=1$. The operators inserted in (1.3) are string operators, which in terms of the Pauli spin operators are $\sigma_{i}^{ \pm}=$ $\left(\sigma_{i}^{x} \pm i \sigma_{i}^{y}\right) / 2$. We consider a generic long-range 1d spin Hamiltonian

$$
\hat{H}_{\mathrm{Gen}}=-\sum_{i} \sum_{j \in \mathbb{Z}} a_{j}\left(\sigma_{i}^{-} \otimes \sigma_{i+j}^{+}\right)+\frac{h}{2} \sum_{i}\left(\sigma_{i}^{z}-\mathbb{I}\right)
$$

where the $a_{j}$ denote arbitrary real coefficients which at least decay as $a_{j} \sim j^{-1-\eta}$ with $\eta>0$. For the application to Chern-Simons theory only exponential decay will be necessary. Our first new result will show that the thermal correlator (1.3) is given by a unitary random matrix model

$$
\begin{aligned}
& F_{j_{1}, \ldots, j_{K} ; l_{1}, \ldots, l_{K}}(\beta)=\frac{\mathrm{e}^{\beta h N}}{(2 \pi)^{N} N !} \int_{-\pi}^{\pi} d \varphi_{1} \ldots \int_{-\pi}^{\pi} d \varphi_{N} \prod_{1 \leq j<k \leq N}\left|e^{i \varphi_{k}}-e^{i \varphi_{j}}\right|^{2}\left(\prod_{j=1}^{N} f_{\beta}\left(e^{i \varphi_{j}}\right)\right) \\
& \times \overline{\hat{s}_{\mu}\left(e^{i \varphi_{1}}, \ldots, e^{i \varphi_{N}}\right)} \hat{s}_{\lambda}\left(e^{i \varphi_{1}}, \ldots, e^{i \varphi_{N}}\right),
\end{aligned}
$$

where the weight function $f_{\beta}\left(e^{i \varphi}\right)$ of the random matrix ensemble is

$$
f_{\beta}\left(e^{i \varphi}\right)=f_{0}\left(e^{i \varphi}\right) \exp \left(\beta \sum_{k \in \mathbb{Z}} a_{k} e^{i k \varphi}\right) .
$$

The second main result of this work follows form a very specific particularization of the first main result and consists in showing that all the elements $S_{\lambda \mu}$ can be recovered as thermal (with $\beta=1$ ) correlation functions of a new 1D Hamiltonian with exponentially decaying interactions, namely:

$$
S_{\lambda \mu}=\left\langle\Uparrow\left|\sigma_{j_{1}}^{+} \cdots \sigma_{j_{N}}^{+} \mathrm{e}^{-\hat{H}_{C S}} \sigma_{l_{1}}^{-} \cdots \sigma_{l_{N}}^{-}\right| \Uparrow\right\rangle
$$

where

$$
\hat{H}_{C S}=-\sum_{i, k \in \mathbb{Z}_{+}} \frac{(-1)^{k+1}}{2 k \sinh \left(\frac{k \gamma}{2}\right)}\left(\sigma_{i}^{-} \otimes \sigma_{i+k}^{+}+\sigma_{i}^{-} \otimes \sigma_{i-k}^{+}\right)+\frac{h}{2} \sum_{i}\left(\sigma_{i}^{z}-\mathbb{I}\right),
$$

$S_{\lambda, \mu}$ are the entries of the $S$-matrix of Chern-Simons theory on $S^{3}$ and gauge group $U(N)$, again $|\Uparrow\rangle$ denotes a ferromagnetic state, characterized by having all of its spins up and $q=\mathrm{e}^{-\gamma}$, since the matrix model formulation that we employ allows to work with the analytical continuation of Chern-Simons theory with $\gamma$ real, which is of relevance in topological string theory [7] and $q$-deformed 2d Yang-Mills theory [22]. The Chern-Simons observables are recovered from the computation of the matrix models, that we identify here with (1.7), by identifying $q=\exp (2 \pi i /(k+N))$. The representations $\lambda$ and $\mu$ are described by Young tableaux [7] and are related to the pattern of flipped spins by $\lambda_{r}=j_{r}-N+r$ and $\mu_{r}=l_{r}-N+r$ [16]. There is freedom in the choice of the magnetic field $h$, which only affects the overall normalization factor in the thermal correlation functions. We shall discuss its role in the context of the framing of the Chern-Simons theory. Note that the rank of the gauge group coincides with the number of 
flipped spins in the thermal correlation function of the spin chain. As particular cases we get the partition function and the quantum dimensions in Chern-Simons theory:

$$
\begin{aligned}
& S_{00}=\langle\ldots, \uparrow, \underbrace{\downarrow, \ldots, \downarrow}_{N}\left|\mathrm{e}^{-\hat{H}_{C S}}\right| \underbrace{\downarrow, \ldots, \downarrow}_{N}, \uparrow, \ldots\rangle, \\
& S_{0 \lambda}=\langle\Uparrow \mid \sigma_{j_{1}}^{+} \cdots \sigma_{j_{K}}^{+} \mathrm{e}^{-\hat{H}_{C S}} \underbrace{\downarrow, \ldots, \downarrow}_{N}, \uparrow, \ldots\rangle .
\end{aligned}
$$

\section{Derivation}

We first prove that the thermal correlators (1.3) associated to the general Hamiltonian (1.4) have the random matrix representation (1.5). The proof will be rigorous by working with a finite chain of $N+1$ sites (and periodic boundary conditions). This is so because the determinantal expression that will be given for the difference-differential equation satisfied by (1.3) will be then unique. The multi-dimensional Riemann sum expression for such a determinant converges then to the unitary matrix model (1.5) in the $N \rightarrow \infty$ limit, which, at the same time, proves that expression (1.3) is well defined in the thermodynamical limit.

The second main result is then a particularization of the first main result, but a very specific one: the coefficients are chosen to be the Fourier coefficients of the logarithm of a theta function. This makes the corresponding generic matrix model to be the one that describes $U(K)$ ChernSimons theory on $S^{3}$.

2.1. Derivation of Main Result 1. We begin by considering the XX Hamiltonian extended to admit generic interactions, denoted by $a_{j}$, to all of its neighbours and on a one-dimensional lattice consisting of $N+1$ sites labeled by elements of the set $M \equiv\{0 \leq k \leq N, k \in \mathbb{Z}\}$, $N+1=0(\bmod 2)$. For simplicity of the presentation we take $N+1$ to be odd and choose the middle site to be indexed by $i=0$. The Hamiltonian is then

$$
\hat{H}_{\mathrm{Gen}}=-\sum_{i=0}^{N} \sum_{j=1}^{(N-1) / 2} a_{j}\left(\sigma_{i}^{-} \otimes \sigma_{i+j}^{+}+\sigma_{i}^{-} \otimes \sigma_{i-j}^{+}\right)+\frac{h}{2} \sum_{i=0}^{N}\left(\sigma_{i}^{z}-\mathbb{I}\right) .
$$

Note that this is the finite chain, with $N+1$ sites, of the Hamiltonian (1.4). We have the following commutation relations

$$
\left[\sigma_{j}^{+}, \hat{H}\right]=-\sigma_{j}^{z} \sum_{i=0}^{(N-1) / 2} a_{i}\left(\sigma_{j+i}^{+}+\sigma_{j-i}^{+}\right)-h \sigma_{j}^{+},
$$

which, reasoning as in [16], give for the $K=1$ correlation function

$$
F_{j l}(\beta) \equiv\left\langle\Uparrow\left|\sigma_{j}^{+} \mathrm{e}^{-\beta \hat{H}_{\mathrm{Gen}}} \sigma_{l}^{-}\right| \Uparrow\right\rangle,
$$

the following differential-difference equation

$$
\frac{d}{d \beta} F_{j l}(\beta)=\sum_{k=1}^{(N-1) / 2} a_{k} F_{j+k, l}(\beta)+\sum_{k=1}^{(N-1) / 2} a_{k} F_{j-k, l}(\beta) .
$$

The generating function associated to these correlators $f_{\beta}(\lambda)=\sum_{j=0}^{N} F_{j l}(\beta) \lambda^{j}$ reads

$$
f_{\beta}(\lambda)=f_{0}(\lambda) \exp \left(\beta\left(\sum_{k=1}^{(N-1) / 2} a_{k} \lambda^{k}+\sum_{k=1}^{(N-1) / 2} a_{k} \lambda^{-k}\right)\right) .
$$


In the $K>1$ case, the same procedure leads for the correlator, in the simplest setting of the XX chain

$$
G_{j_{1}, \ldots, j_{K} ; l_{1}, \ldots, l_{K}}(\beta)=\left\langle\Uparrow\left|\sigma_{j_{1}}^{+} \cdots \sigma_{j_{K}}^{+} \mathrm{e}^{-\beta \hat{H}_{X X}} \sigma_{l_{1}}^{-} \cdots \sigma_{l_{K}}^{-}\right| \Uparrow\right\rangle,
$$

it simply satisfies a multi-index version of the difference-differential equation for the $K=1$ case. In particular

$$
\frac{d}{d \beta} G_{j_{1}, \ldots, j_{K} ; l_{1}, \ldots, l_{K}}(\beta)=\frac{1}{2} \sum_{l=1}^{K}\left(G_{j_{1}, \ldots, j_{l+1}, \ldots, j_{K} ; l_{1}, \ldots, l_{K}}(\beta)+G_{j_{1}, \ldots, j_{l-1}, \ldots, j_{K} ; l_{1}, \ldots, l_{K}}(\beta)\right)
$$

As pointed out in [23], the solution to this equation, as can be easily checked explicitly, can be written as a determinant whose entries satisfy the $K=1$ differential-difference equation. The key point is that the same result holds for the generalization considered here. In particular, in our case, we have that, for $K \leq N$

$$
\frac{d}{d \beta} F_{j_{1}, \ldots, j_{K} ; l_{1}, \ldots, l_{K}}(\beta)=\frac{1}{2} \sum_{k=1}^{(N-1) / 2} \sum_{l=1}^{K} a_{k} F_{j_{1}, \ldots, j_{l}+k, \ldots, j_{K} ; l_{1}, \ldots, l_{K}}(\beta)+\sum_{k=1}^{(N-1) / 2} \sum_{l=1}^{K} a_{k} F_{j_{1}, \ldots, j_{l}+k, \ldots, j_{K} ; l_{1}, \ldots, l_{K}}(\beta),
$$

and the determinantal form for the correlator

$$
F_{j_{1}, \ldots, j_{K} ; l_{1}, \ldots, l_{K}}(\beta)=\operatorname{det}\left(F_{j_{r} l_{s}}\right)_{r, s=1}^{K}
$$

satisfies (2.5), if the $F_{j_{r} l_{s}}$ satisfy (2.3), as can be easily checked explicitly. The Fourier coefficients of the generating function contain the correlators. In the $K=1$ and for a finite $N$ chain, they read

$$
F_{j l}(\beta)=\frac{1}{N+1} \sum_{s=0}^{N} \exp \left(\beta\left(\sum_{k=1}^{(N-1) / 2} a_{k} e^{i k \theta_{s}}+\sum_{k=1}^{(N-1) / 2} a_{k} e^{-i k \theta_{s}}\right)\right) e^{i \theta_{s}(j-l)}
$$

where $\theta_{s}=\frac{2 \pi}{N+1}\left(s-\frac{N}{2}\right)$. Notice that this extends the result in [24] to the case of arbitrary number of neighbour interactions. Likewise, the multi-dimensional Fourier coefficient is then, with the same expression for $\theta_{s}$ as above with $s=0,1, \ldots N$, as in [24]

$$
\begin{aligned}
F_{j_{1}, \ldots, j_{K} ; l_{1}, \ldots, l_{K}}(\beta)= & \frac{1}{(N+1)^{K}} \sum_{N \geq s_{1}>s_{2}>\ldots>s_{K} \geq 0} \exp \left(\beta \sum_{l=1}^{K}\left(\sum_{k=1}^{(N-1) / 2} a_{k} e^{i k \theta_{s_{l}}}+\sum_{k=1}^{(N-1) / 2} a_{k} e^{-i k \theta_{s_{l}}}\right)\right) \\
& \times \prod_{1 \leq j<k \leq K}\left|e^{i \theta_{k}}-e^{i \theta_{j}}\right|^{2} \overline{\hat{s}_{\mu}\left(e^{i \theta_{1}}, \ldots, e^{i \theta_{K}}\right)} \hat{s}_{\lambda}\left(e^{i \theta_{1}}, \ldots, e^{i \theta_{K}}\right) .
\end{aligned}
$$

This analytical expression can also be understood to follow immediately from the Toeplitz minor identity for determinants of the type (2.6), given in [25]. In this discrete random matrix representation of the correlator, $\hat{s}_{\lambda}\left(e^{i \varphi}, \ldots, e^{i \varphi}\right)$ is a Schur polynomial [25] and the representation $\lambda$ is indexed by a partition $\left\{\lambda_{i}\right\}_{i=1}^{N}$ which is related to the sequence $\left\{j_{i}\right\}_{i=1}^{N}$ in the spin operators by $\lambda_{r}=j_{r}-N+r$ [26]. The other sequence in the correlator is correspondingly accounted for by the other Schur polynomial in (2.7).

By taking the slowest decay of the coefficients to be $a_{j} \sim j^{-1-\eta}$ with $\eta>0$, the limit of an infinite spin chain $N \rightarrow \infty$ renders these Riemann sums into their corresponding integral form

\footnotetext{
${ }^{1}$ The $N+1$ is choosing the convention of Bogoliubov et al where of $N+1$ sites labeled by elements of the set $M \equiv\{0 \leq k \leq N, k \in \mathbb{Z}\}, N+1=0(\bmod 2)$ [24]. See also 24] for the Riemann sum expression which corresponds to the XX Hamiltonian.
} 
[27, leading to a $U(K)$ unitary random matrix ensemble

$$
\int_{-\pi}^{\pi} d \varphi_{1} \ldots \int_{-\pi}^{\pi} d \varphi_{K} \prod_{1 \leq j<k \leq K}\left|e^{i \varphi_{k}}-e^{i \varphi_{j}}\right|^{2}\left(\prod_{j=1}^{K} f_{\beta}\left(e^{i \varphi_{j}}\right)\right) \overline{\hat{s}_{\mu}\left(e^{i \varphi_{1}}, \ldots, e^{i \varphi_{K}}\right)} \hat{s}_{\lambda}\left(e^{i \varphi_{1}}, \ldots, e^{i \varphi_{K}}\right),
$$

2.2. Derivation of Main result 2. The next step is to recall from [28, 29] that $U(N)$ ChernSimons theory on $S^{3}$ can be described in terms of a unitary matrix model. In particular, the matrix model is of the type (2.8) but with a weight function

$$
f(\varphi)=\Theta_{3}\left(\mathrm{e}^{\mathrm{i} \varphi_{j}} \mid q\right)=\sum_{n=-\infty}^{\infty} \mathrm{q}^{n^{2} / 2} \mathrm{e}^{i n \varphi}
$$

which is Jacobi's third theta function.

Hence, to reproduce Chern-Simons theory we have to consider the generalized spin chain (2.1) in the specific setting where the resulting generating function (2.4) turns out to be the theta function (2.9). The Fourier coefficients of $\ln \Theta_{3}\left(\mathrm{e}^{\mathrm{i} \varphi_{j}} \mid q\right)$ can be easily obtained by using the product form of (2.9) given by the Jacobi triple product identity

$$
\Theta_{3}\left(\mathrm{e}^{\mathrm{i} \varphi} \mid q\right)=\prod_{j=1}^{\infty}\left(1-q^{j}\right)\left(1+q^{j-1 / 2} \mathrm{e}^{\mathrm{i} \varphi}\right)\left(1+q^{j-1 / 2} \mathrm{e}^{-\mathrm{i} \varphi}\right) .
$$

Taking the logarithm leads to

$$
\log \Theta_{3}\left(\mathrm{e}^{\mathrm{i} \varphi} \mid q\right)=\sum_{j=1}^{\infty} \log \left(1-q^{j}\right)+\sum_{j=1}^{\infty}\left(\log \left(1+q^{j-1 / 2} z\right)+\log \left(1+q^{j-1 / 2} / z\right)\right) .
$$

The first term is the Fourier coefficient of order zero. We discuss its relevance below and focus on the non-trivial part of (2.11). Using the Taylor expansion of $\log (1+x)$, we have

$$
\begin{aligned}
\sum_{j=1}^{\infty}\left(\log \left(1+q^{j-1 / 2} z\right)+\log \left(1+q^{j-1 / 2} / z\right)\right) & =\sum_{j=1}^{\infty} \sum_{n \in \mathbb{Z}, n \neq 0}^{\infty} \frac{(-1)^{n+1}}{n} q^{(j-1 / 2) n} z^{n} \\
& =\sum_{n \in \mathbb{Z}, n \neq 0}^{\infty} \frac{(-1)^{n+1}}{n} z^{n} \sum_{j=1}^{\infty} q^{n(j-1 / 2)} \\
& =\sum_{n \in \mathbb{Z}, n \neq 0}^{\infty} \frac{(-1)^{n+1} q^{n / 2}}{n\left(1-q^{n}\right)} z^{n} .
\end{aligned}
$$

Therefore, the Fourier coefficient $a_{n}$ of order $n(n \in \mathbb{N})$, reads

$$
a_{n}=a_{-n}=\frac{(-1)^{n+1} q^{n / 2}}{n\left(1-q^{n}\right)},
$$

and we have that

$$
a_{k}=\frac{(-1)^{k+1}}{2 k \sinh \left(\frac{k \gamma}{2}\right)}
$$

with $k \in \mathbb{Z}$ and with $q=\exp (-\gamma)$ the $q$-parameter of Chern-Simons theory. In addition, we have to choose $\beta=1$ in (2.4) because plugging (2.13) in (2.4) gives $\Theta_{3}^{\beta}\left(\mathrm{e}^{\mathrm{i} \varphi_{j}} \mid q\right)$. The orthogonality of the states fixes the initial condition for $\beta=0$ to be $\delta_{j, l}$ and this implies that $g_{0}(\lambda)=1$. This finishes the proof of the main result.

Notice that the Fourier coefficient of order zero, which is $a_{0}=N \sum_{n=1}^{\infty} \ln \left(1-q^{n}\right)$ has not been included. It only contributes with a normalization factor and hence can be taken into account by fixing the value of the external magnetic field. In any case, this choice of normalization gives an 
exact correspondence with the unitary matrix model with a theta function [30, 29]. Its partition function is explicitly given below where we also explain that framing can be taken into account by a proper choice of the magnetic field.

\section{Discussion}

A recent result [31] shows that the error obtained by approximating (2.8) by its Riemann sum decreases exponentially with $L$. More concretely, the relative error is $\mathcal{O}\left(e^{-c(L-N)}\right)$ as $L-N \rightarrow \infty$, even if $N$ also goes to $\infty$. This result holds for Toeplitz determinants of discrete measure under very general conditions and it immediately applies to $S_{00}$. The same result is expected to hold for $S_{\mu \lambda}$ because due to (1.7) and (2.8) we know it is the minor of a Toeplitz matrix [25. These results suggest that the quantum topology of a manifold can be described with a finite ring of spins interacting as explained above 2 .

The finite chain case is also of special interest in its own right because the discretization of the matrix model also emerges naturally in the study of Chern-Simons theory on $S^{2} \times S^{1}$ with matter in the fundamental representation of the gauge group (vector matter). In recent work [32, it has been shown that, in the large $N$ limit, the observables of that theory are given by a discrete unitary matrix model with a certain potential $V(U)=T^{2} V_{2} v(U)$, where $T$ is the temperature (inverse of the radius of $S^{1}$ ), $V_{2}$ is the volume of $S^{2}$ and $v(U)$ is the potential that has to be computed, in an effective field theory approach, for every theory (that is, depends on the choice of matter made) 32 .

Thus, the matrix model description is precisely the one that holds for, more realistic, finite spin chains. Notice that the potential now is not $\ln \theta_{3}\left(\mathrm{e}^{i \varphi}, q\right)$ in general. This simply means that other choices for the coefficients $a_{k}$ should be made, but, as we have seen, any system characterized by a well-defined unitary matrix model with single trace terms $c_{k} \operatorname{Tr} U^{k}$ with $k \in \mathbb{Z}$ in the potential is described in the same manner by the corresponding spin chain model.

It is also interesting to explore how robust are the quantum topological properties of the spin chain. Namely, is the interaction chosen in (1.8) unique? In particular, for the ChernSimons unitary ensemble, it is known that $g(\varphi)=\theta_{3}^{-1}\left(-\mathrm{e}^{i \varphi}, q\right)$ is also a valid weight function for the matrix model to describe Chern-Simons theory [33]. In our context, this implies that we can modify the interactions in (1.8) with an alternating ferromagnetic-antiferromagnetic coupling. If one considers, as above, the Fourier coefficients of $\ln g(\varphi)=-\ln \theta_{3}\left(-\mathrm{e}^{i \varphi}, q\right)$, then the corresponding Fourier coefficients (2.13) are modified accordingly to

$$
b_{k}=\frac{1}{2 k \sinh \left(\frac{k \gamma}{2}\right)},
$$

and the resulting spin chain

$$
\hat{H}_{C S}=-\sum_{i, k \in \mathbb{Z}_{+}} \frac{1}{2 k \sinh \left(\frac{k \gamma}{2}\right)}\left(\sigma_{i}^{-} \otimes \sigma_{i+k}^{+}+\sigma_{i}^{-} \otimes \sigma_{i-k}^{+}\right)+\frac{h}{2} \sum_{i}\left(\sigma_{i}^{z}-\mathbb{I}\right),
$$

possesses the same topological properties. Notice that, in the expression given above (Main result 2) we are led to an alternating ferromagnetic-antiferromagnetic interaction but with the same interaction coefficients otherwise. However, the first and leading interaction is in both cases ferromagnetic. This equivalence of both spin chain models is exact for the partition function $S_{00}$ [33], which is given by a matrix model without any insertion of a Schur polynomial. Regarding the quantum dimensions or the full topological S-matrix, the alternative choice (3.1) gives the same observables but with transposed partitions $\lambda^{\prime}, \mu^{\prime}$ (the columns of the Young tableaux

\footnotetext{
${ }^{2}$ The discussion on experimental accessibility made in [16] for the XX-model applies also here.
} 
becomes rows and conversely). Thus, alternatively, if one wants equality of the correlators the respective pattern of flipped spins is different (but immediately related) depending on the election of (2.13) or (3.1).

Notice that there is also freedom in the choice of the external magnetic field since ChernSimons theory is actually a theory of framed knots and links. Framing $\Pi$ on a three-manifold $M$ (here $M=S^{3}$ ), is a choice of trivialization of the bundle $T M \oplus T M$ [34. It contributes multiplicatively to the observables and for gauge group $G$ its explicit expression is 30]

$$
\delta(M, \Pi)=\exp \left(\frac{2 \pi \mathrm{i} s}{24} c\right)=\exp \left(\frac{\pi \mathrm{i} s\left(N^{2}-1\right) k}{(k+N)}\right),
$$

where $c$ is the central charge of the associated Wess-Zumino-Witten model based on the affine extension of $G$. Notice that it is parametrized by an integer $s \in \mathbb{Z}$. In the second expression we have particularized for the case $G=U(N)$. At the level of anyon physics, the framing contribution in the Chern-Simons theory describes how anyons rotate while they wind around each other [35].

For example with the choice $h=\sum_{n=1}^{\infty} \ln \left(1-q^{n}\right)$ to account for the zeroth order Fourier coefficients of the potential, the correlator is exactly the theta function matrix model, whose partition function is exactly [29]

$$
\langle\ldots, \uparrow, \underbrace{\downarrow, \ldots, \downarrow}_{N}\left|\mathrm{e}^{-\hat{H}_{C S}}\right| \underbrace{\downarrow, \ldots, \downarrow}_{N}, \uparrow, \ldots\rangle=\mathrm{e}^{-i \pi N(N+1)} \prod_{i=1}^{N-1}\left(1-q^{i}\right)^{N-i} .
$$

To account for the framing contribution (3.3) one can simply add the corresponding term in the magnetic field $h$ taking into account the general result (2.8).

\section{OpEN PROBLEMS}

There are several interesting open questions. First, to what extent finite spin chains can be engineered to reproduce the observables of a Chern-Simons theory with matter. This theory is no longer topological and the degeneracy at zero energy of Chern-Simons theory is broken. It would be interesting to understand if the finite size of the spin chain model achieves this in a natural way. Second, we have seen that the XX models extended with additional interactions have remarkable properties from the point of view of the theory of exactly solvable systems since its correlation functions have an immediate connection with the WZW model and admit very wellknown exact expressions for every $N$, something that does not hold in the case of the ordinary XX model. Thus, it would be interesting now to study the properties of the Hamiltonian, including spectral properties and the establishment of its universality class, which is expected to be the same as that of the XX model, due to the very fast decay of the additional interactions. Comparison with other exactly solvable spin chains with infinitely many interactions such as the Inozemtsev model [36] or the Richardson-Gaudin models would be also of interest.

To extend the discussion to the case of $q$ root of unity is another interesting open problem. The type of series expansions involved in the discussion of Chern-Simons theory are $q$-series, which happen to be much better known for $q$ real and $q<1$ (or $q>1$ ). Indeed, these series are analytic inside $|q|<1$ and they have $|q|=1$ as a natural boundary. Notice for example also that for $q$ root of unity the power series coefficients above are not even defined, due to the appearance of terms such as the $q$-Pochhammer symbol $(q ; q)_{n}:=\prod_{j=0}^{n-1}\left(1-q^{j}\right)$. In spite of this, it can be shown that most properties holds in that case as well [38], and would be interesting to take these results into account to reproduce the construction here for $q$ root of unity. Furthermore, these more delicate analysis of the $q$-series may lead to further understanding of the model. For 
example, a well-known identity

$$
\sum_{n=0} \frac{z^{n}}{(q ; q)_{n}}=\exp \left(\sum_{n=1}^{\infty} \frac{z^{n}}{n\left(1-q^{n}\right)}\right),
$$

was shown by Hardy and Littlewood to hold, even for $q$ root of unity [38]. One could then use (4.1) and similar properties [38] to extend our result to $q$ roots of unity.

However, this needs to be thoroughly investigated, both for $q$ real and $q$ root of unity. Another clear open problem is to extend this description to the case of knot and link polynomials invariants. Very recently it has been shown that a number of colored HOMFLY polynomial invariants can be written as a terminating basic hypergeometric function [37. A possible way to relate this to the results presented here is the fact that these functions admit an integral representation, which in turn can be also, in principle, identified with a unitary matrix model. This is left as an open problem, to be discussed elsewhere.

Acknowledgements. We thank Belén Paredes and Germán Sierra for discussions at initial stages of this work and Milosz Panfil for comments. The work of MT was concluded while starting as Investigador FCT at Universidade de Lisboa. Both authors acknowledge support from MINECO (grant MTM2011-26912), Comunidad de Madrid (grant QUITEMAD+-CM, ref. S2013/ICE-2801) and the European CHIST-ERA project CQC (funded partially by MINECO grant PRI-PIMCHI-2011-1071). This work was also made possible through the support of grant \#48322 from the John Templeton Foundation. The results obtained in this publication are those of the authors and do not necessarily reflect the views of the John Templeton Foundation.

\section{Appendix A. Definitions}

We introduce some Lie algebra conventions and definitions, focussing on $U(N)$. We use the standard notations as in [18] $\omega_{i}$ for $i=1, \ldots, N$, denotes the set of fundamental weights, and $\mathrm{e}_{i}$ are unit vectors in $\mathbb{R}^{N}$. The set of fundamental weights is then defined by

$$
\omega_{i}=\sum_{j=1}^{i} \mathrm{e}_{j}-\frac{i}{N} \sum_{j=1}^{N} \mathrm{e}_{j} .
$$

There is also the notion of simple roots, which are given by $\alpha_{i}=\mathrm{e}_{i}-\mathrm{e}_{i+1}$ for $i=1, \ldots, N-1$. One can expand the highest weight of an irreducible representation

$$
\lambda=\sum_{i=1}^{N} \lambda_{i} \omega_{i}=\sum_{i=1}^{N}\left(\ell_{i}-\kappa\right) \mathrm{e}_{i}
$$

where

$$
\kappa=\frac{1}{N} \sum_{j=1}^{N} j \ell_{j}
$$

is the number of boxes in the Young tableaux divided by $N$, and

$$
\ell_{i}=\lambda_{i}+\lambda_{i+1}+\ldots+\lambda_{i+N} .
$$

With these definitions, it is immediate to check that (A.2) holds. The Weyl chamber is a connected set in $\mathbb{R}^{N}$ left out after we delete all hyperplanes orthogonal to the roots. Its definition is

$$
C_{\omega}=\left\{\lambda\left(\omega \lambda, \alpha_{i}\right) \geq 0, i=1, \ldots N-1\right\}, \omega \in W,
$$

where $W$ denotes the Weyl group. The fundamental chamber mentioned in the Introduction corresponds to the identity element, $C_{0}$. It is manifest from the definition that $\lambda_{i} \geq 0$ if $\lambda$ is 
in the fundamental chamber and, therefore, the $\ell^{\prime} s$ satisfy $\ell_{i} \geq \ell_{i+1}$ and one can define the coordinates:

$$
h_{i}=\ell_{i}+\frac{N+1}{2}-i .
$$

The fundamental chamber then is the domain $h_{1}>h_{2}>\ldots>h_{N}$. These are the variables that are usually used to label representations, and which we have labeled by $\lambda$. They can be written as

$$
h=\ell+\rho
$$

where $\rho$ is the Weyl vector $\rho:=\sum_{i=1}^{N} \omega_{i}$

This explains the notations in the Introduction and how the states of the Hilbert space, denoted by $|\lambda\rangle$, and which are integrable representations, can be indexed, at the practical level, by Young tableaux.

\section{REFERENCES}

[1] D. Birmingham, M. Blau, M. Rakowski and G. Thompson, Phy. Rep. 209, (1991) 129-340.

[2] E. Witten, Commun. Math. Phys. 121, 351 (1989).

[3] S. C. Zhang, T. H. Hansson and S. Kivelson, Phys. Rev. Lett. 62, 82-85 (1989).

[4] C. Nayak et al., Rev. Mod. Phys. 80, 1083 (2008)

[5] J. K. Pachos, Introduction to Topological Quantum Computation, Cambridge University Press (2012).

[6] G. K. Brennen and J. K. Pachos, Proceed. Roy. Soc. A 464, 1-24 (2008)

[7] M. Marino, Rev. Mod. Phys. 77, 675 (2005), [hep-th/0406005]

[8] J. I. Cirac, P. Maraner, J. K. Pachos, Phys. Rev. Lett. 105190403 (2010)

[9] K. Temme, T. J. Osborne, K. G. Vollbrecht, D. Poulin, and F. Verstraete, Nature 471, 87 (2011)

[10] S. P. Jordan, K. S. M. Lee and J. Preskill, Science 336, 1130 (2012)

[11] M. Lewenstein et al., Adv. Phys. 56, 243 (2007)

[12] M. Lewenstein, A. Sanpera and V. Ahufinger, Ultracold Atoms in Optical Lattices: Simulating quantum many-body systems, Oxford University Press, (2012).

[13] L. Tagliacozzo et al., Ann. Phys. 330, 160 (2013)

[14] D. Banerjee et al., Phys. Rev. Lett. 110, 125303 (2013).

[15] E. Zohar, J. I. Cirac and B. Reznik, Phys. Rev. Lett. 110, 055302 (2013); Ibid. 125304

[16] D. Pérez-García and M. Tierz, Phys. Rev. X 4, no. 2, 021050 (2014) [arXiv:1305.3877 [cond-mat.str-el]].

[17] V. Jones, Ann. of Math. 126, 335 (1987).

[18] P. Di Francesco, P. Mathieu, and D. Sénéchal, Conformal Field Theory, Springer-Verlag (1997).

[19] W.B.R. Lickorish, An Introduction to Knot Theory, Springer-Verlag (1997)

[20] V. Turaev, Quantum Invariants of Knots and 3-Manifolds, De Gruyter Studies in Mathematics (2010)

[21] F. A. Bais, J. C. Romers, New J. Phys. 14, 035024 (2012), [arXiv:1108.0683]

[22] R. J. Szabo and M. Tierz, Nucl. Phys. B 876, 234 (2013) [arXiv:1305.1580 [hep-th]].

[23] N. M. Bogoliubov, J. Math. Sci. 138, 5636 (2006).

[24] N.M. Bogoliubov and C. Malyshev, St. Petersburg Math. Jour. 22, 359 (2011).

[25] D. Bump and P. Diaconis J. Comb. Theor. A97, 252 (2002)

[26] N. M. Bogoliubov, A.G. Pronko and J. Timonen, Zap. Nauchn. Sem. POMI, 403, 5-18 (2012), arXiv:1102.5639.

[27] J. J. Ruch and M. Weber, Note di Matematica 26, n. 2, 1-50, (2006).

[28] T. Okuda, J. High Energy Phys. 0503 (2005) 047 [arXiv:hep-th/0409270]|

[29] M. Romo and M. Tierz, Phys. Rev. D 86 (2012) 045027[[arXiv:1103.2421 [hep-th]]

[30] R. J. Szabo and M. Tierz, J. Phys. A 43 (2010) 265401 [arXiv:1003.1228 [hep-th]]

[31] J. Baik and Z. Liu, Int. Math. Res. Not. (2013) [arxiv:1212.4467]

[32] S. Jain, S. Minwalla, T. Sharma, T. Takimi, S. R. Wadia and S. Yokoyama, [arXiv:1301.6169 [hep-th]]

T. Takimi, JHEP 07, 177, (2013), [arXiv:1304.3725 [hep-th]]

[33] R. J. Szabo and M. Tierz, J. Math. Phys. 53 (2012) 103502 [arXiv:1005.5643 [hep-th]]

[34] M. F. Atiyah, Topology 29, 1 (1990).

[35] M. Freedman et al., Annals of Physics 310 (2004) 428-492

[36] V.I. Inozemtsev, Lett. Math. Phys. 17 (1989) 11

[37] G. Giasemidis and M. Tierz, Lett. Math. Phys. 104, 1535 (2014) [arXiv:1401.8171 [hep-th]]. 
[38] D. S. Lubinsky, J. Number Theor., 76 (1999), 217-247.

Departamento de Análisis Matemático and Instituto de Matemática Interdisciplinar, Facultad de Ciencias Matemáticas, Universidad Complutense de Madrid, 28040 Madrid, Spain

E-mail address: dperez@mat.ucm.es

Departamento de Matemática, Grupo de Física Matemática, Faculdade de Ciências, Universidade de Lisboa, Campo Grande, Edifício C6, 1749-016 Lisboa, Portugal.

E-mail address: tierz@fc.ul.pt

Departamento de Análisis Matemático, Facultad de Ciencias Matemáticas, Universidad ComPlutense De MAdRID, 28040 MAdrid, SPAin

E-mail address: tierz@mat.ucm.es 